\title{
Extraction of Three-Dimensional Information of Biological Membranous Tissue with Scanning Confocal Infrared Laser Microscope Tomography
}

\author{
Soonwook Kwon, ${ }^{1}$ Se-Bum Choi, ${ }^{2}$ Min Gyu Park, ${ }^{2}$ Hyunung Yu, ${ }^{2, \star}$ Seung-Woo Suh, ${ }^{3}$ \\ and Im Joo Rhyu ${ }^{1, *}$ \\ ${ }^{1}$ Department of Anatomy, Korea University College of Medicine, Seoul 136-705, Korea \\ ${ }^{2}$ Korea Research Institute of Standards and Science, Daejon 305-340, Korea \\ ${ }^{3}$ Department of Orthopedics, Korea University Guro Hospital, Seoul 152-703, Korea
}

\begin{abstract}
The "LEXT" confocal laser scanning microscope has been used for the three-dimensional (3D) imaging of the surface of specimens, especially in materials science fields, by the penetration of near-infrared (NIR) light without mechanical cutting, deposition, or other specimen pretreatment. Noninvasive investigation of various biological tissues such as human spinal dura mater, rat aorta, and cornea without the dehydration process was successfully carried out with the "LEXT," in order to access both surface and internal topographic images of the biological structures at a good status of the wet tissue such as in vivo, especially in measuring tissue thickness. The confocal NIR laser microscopy offers the viable means to visualize tissue architecture and its thickness in microdomain to integrate 3D images efficiently. We believe that the "LEXT" has a good application for biological researchers to study biomaterials, and it would be useful as a diagnostic tool in the near future.
\end{abstract}

Key words: laser microscope, imaging, confocal, infrared, scanning, dura mater, membrane

\section{INTRODUCTION}

The development of various microscopic systems has significantly contributed to the advances in sciences, especially in the field of biological and materials sciences. Invention of the light microscope opened new biological fields such as histology and microbiology at the end of the 16th century. Moreover, the microscopic technique including histological preparation techniques and optics accelerated the development of biological sciences (Clay \& Court, 1975; Heinrichs, 2009). By the introduction of an electron beam to overcome the resolution limit of the light microscope, the first $17.4 \times$ magnification power (Ruska, 1987) has increased to observe small molecules up to the atomic scale nowadays.

Recently, the confocal near-infrared (NIR) laser scanning microscope (LEXT) has been introduced in the field of materials science as a three-dimensional (3D) topographical imaging tool for surface imaging of specimens without physical microsectioning, deposition, or other pretreatments of the specimen. Because of the low scattering at a long wavelength, the "LEXT" has a good, efficient penetration power of up to $500 \mu \mathrm{m}$ depth.

Many biological tissues have a membranous nature, whose thickness is much related to the normal physiological function and diagnostic criteria in some clinical diseases. For a microscopic evaluation of biological tissue, histologi$\mathrm{cal}$ and conventional tissue preparation steps including fixation, dehydration, embedding, sectioning, and staining are required before microscopic observation. These processes are quite elaborate and time consuming, and lead to an

() MICROSCOPY SOCIETY OF AMERICA 2013

${ }^{\star}$ Corresponding authors. E-mail: peacewithu@kriss.re.kr; irhyu@korea.ac.kr inevitable alteration of the tissue in the meantime, such as shrinkage or distortion of samples.

In this study, we introduce a novel imaging experiment of biological specimen with the "LEXT" instrument, free from shrinkage artifact and long-term tissue processing time, to obtain unique 3D information including virtual microsectional images and tissue thickness close to wet tissue sample in vivo.

\section{Materials and Methods}

\section{Preparation of the Human Dura Mater}

A cadaver for medical student education with no history of spinal surgery or deformity was obtained under Korea University Anatomical Donation Program and treated with an accurate observance of the university policy. A 70-year-old male cadaver was selected. It was perfused through the femoral artery with $10 \%$ neutral formalin for complete fixation. The lumbar vertebral level (L2-L3, posterior dura mater) after laminectomy was chosen to be fixed in a $10 \%$ neutral formalin bottle in order to preserve the thickness and structure.

\section{Preparation of the Cornea, Aorta, and Pericardium of Sprague-Dawley Rat}

Sprague-Dawley male rats (14 weeks old) were anesthetized with sodium pentobarbital $(100 \mathrm{mg} / \mathrm{kg})$. Then, the thorax was opened and perfused through the left ventricle of the heart with $4 \%$ paraformaldehyde in $0.1 \mathrm{M}$ Millonig's buffer ( $\mathrm{pH}$ 7.4). The pericardium was obtained very carefully after perfusion. The right cornea and the descending aorta of the thorax were excised with fine scissors. To observe the central 

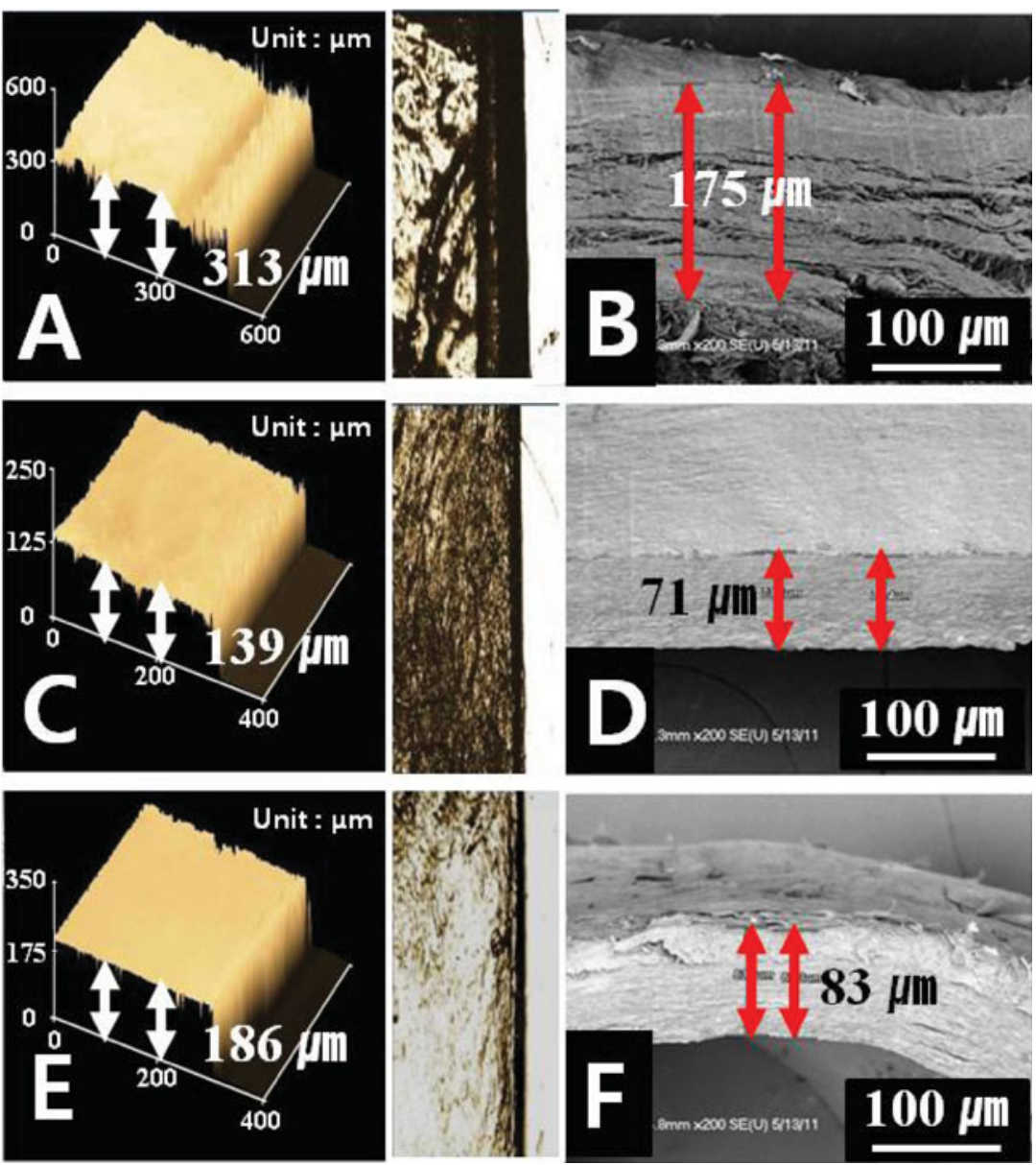

Figure 1. Representative three-dimensional (3D) tomographical image of the biological tissue, whose thickness is measured with the "LEXT" by a distance between the top and the bottom (left, 3D and top view images) and SEM (right): (A, B) the human dura mater, $(\mathbf{C}, \mathbf{D})$ the rat aorta; $(\mathrm{E}, \mathrm{F})$ the rat cornea. part of the cornea, it was cut using a fine blade. The midline of the aorta was cut like a spread cylinder to make it unfolded and flat.

\section{Observation by the Scanning Confocal NIR Laser Microscope}

3D microscopic imaging processes of various tissues were carried out with the LEXT (NIR, 1,310 nm) microscope equipped with a confocal diaphragm (LEXT OLS3000-IR; Olympus, Japan) and an InGaAs photodetector after a pinhole to eliminate out-of-focus images. The $x, y$ frame image was reconstructed in a subsecond with a piezo scanner to observe the internal structure. A $z$-axial scan was also performed for each sectional plane with a 2- $\mu \mathrm{m}$-depth resolution. After a 3D scan was completed, manual pointing for thickness (distance between the bottom and the top of the specimen) was performed (Fig. 1).

\section{Scanning Electron Microscopy (SEM, Hitachi S-4700)}

The samples were pre fixed with $2.5 \%$ glutaraldehyde and $4 \%$ paraformaldehyde at $4^{\circ} \mathrm{C}$ overnight, and then washed with phosphate buffer. Postfixation with $1 \%$ osmium tetroxide for 30 min was performed, followed by dehydration in a routine order with EtOH and then with $t$-butyl alcohol. Samples were coated with Pt.

\section{Results}

\section{The Thickness of Biological Tissues}

We measured the thickness of the wet sample with the "LEXT" microscope and then compared a dehydrated tissue with SEM evaluation to figure out the methodological difference. The "LEXT" program could make a 3D square block by surface rendering from the top view (Figs. 1A, 1C, $1 \mathrm{E})$, whereas only lateral images in the SEM measurement (200× magnification) were captured to deduce the thickness from the interval in between (Figs. 1B, 1D, 1F). The values observed with the LEXT are quite comparable to the size of the lateral section of the wet tissue obtained with a conventional optical microscope (Fig. 2A), whereas the values from SEM are a half time thinner than those by "LEXT." For example, the human dura mater showed $313 \mu \mathrm{m}$ in the "LEXT" (Fig. 1A) and $175 \mu \mathrm{m}$ in the SEM (Fig. 1B).

\section{Virtual Sections of Biological Tissues by the "LEXT"}

Rapid-scan images of the tissue morphology in situ by regular steps along the $z$-axis were integrated to calculate the full thickness of the tissue (Fig. 1). Along the vertical axis in Figure 2A, the characteristic architecture of the virtual section at each level was shown (Fig. 2B). The internal architecture at each focal plane showed branched 

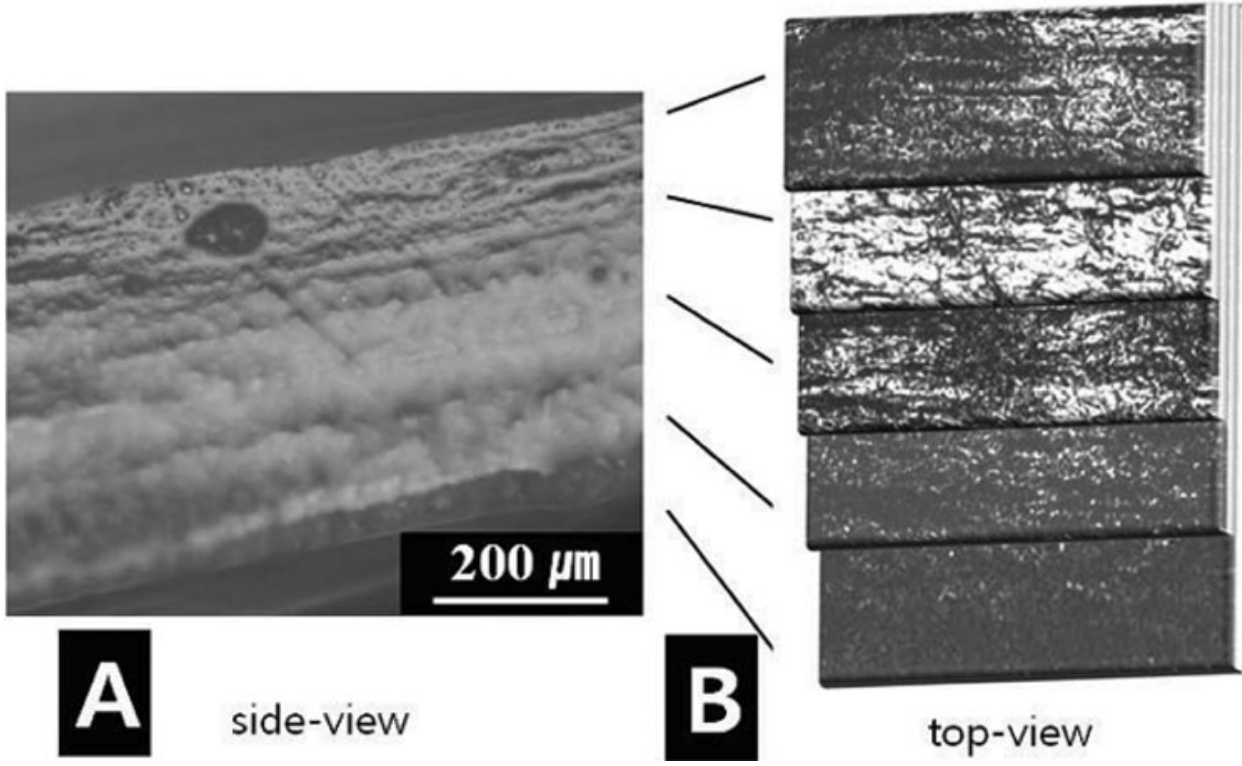

Figure 2. A side view with an optical microscope (A) and the corresponding top view transverse images (B) of the human dura mater showing a series of virtual-sectional architectures.

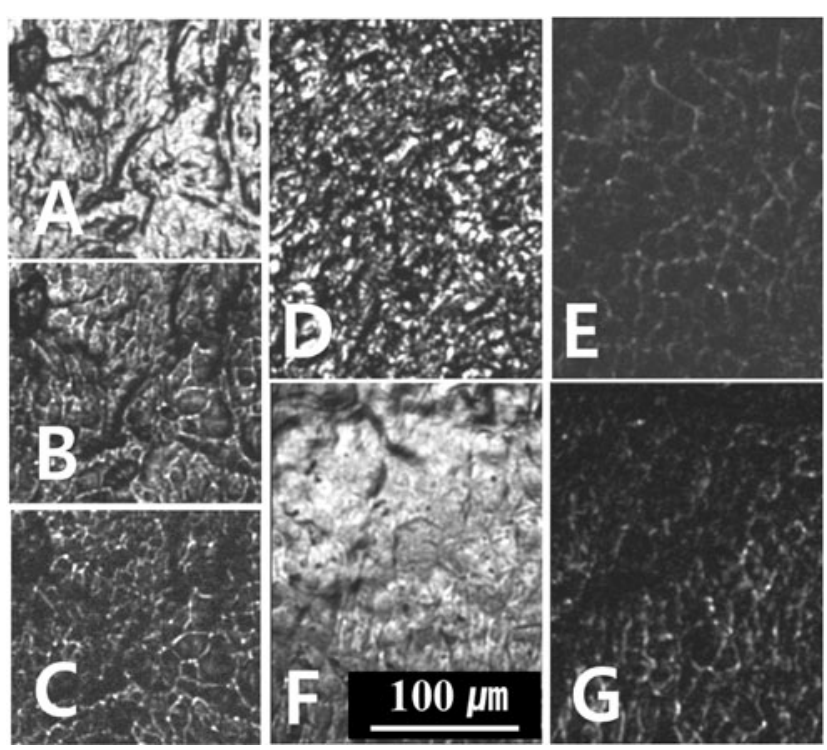

Figure 3. Representative tissue images of the human dura mater captured from the top along the $z$-axis by $10-\mu \mathrm{m}$-step in a row $(\mathrm{A}-\mathrm{C})$, and various internal architectures are shown according to the nature of the tissues ( $20 \times$ objective lens); (D) the rat aorta; (E) endothelium of the rat cornea; (F) stroma of the rat cornea; (G) epithelium of the rat cornea.

grooves (a capillary network), which divided the tissue into several compartments (Figs. 3A-3C). The polygonal-type network was demarcated by a white signal observed on each virtual section (Figs. 3A-3C). The white signal appears to be a tiny network composed of connective tissue.

In addition, rat aorta and cornea were observed (Figs. 3D-3G). The network of grooves was also observed on the rat aorta as in the human dura mater, which might be vasa vasorum, and clusters of white signals were scattered in the black background (Fig. 3D). The virtual section images of the cornea revealed three distinct features from the surface layer to inner layers (Figs. 3E-3G). The honeycomb- like structure was observed in the endothelium of the cornea (Fig. 3E), followed by a homogenous stromal layer (Fig. 3F). Honeycomb-like structures that were relatively smaller than that of the epithelium were observed on the inner layer, which seemed like the endothelium (Fig. 3G).

\section{DiscusSION}

Previous studies used a pseudoconfocal mode such as a slit scanning confocal microscope with in vivo observation (Jalbert et al., 2003). Our instrument with a conjugate pinhole provides a true confocal geometry and a rapid scan, typically several image frames per second, with the aid of a galvanomirror attached to an MEMS scanner. A highperformance computer was used to reconstruct the image in order to visualize it in real time (Mahapatro et al., 2009).

This study suggested a useful application of the "LEXT" to measure biological membranous tissues. The measured thickness of the tissues with the "LEXT" was 1.8-2.2 times thicker than that of the SEM preparation because of the dehydration process (Fig. 1). Comparative measurement of the human ear drum with confocal microscopy and conventional microscopy showed 30\% shrinkage during tissue preparation (Kuypers et al., 2006). These data indicate that the degree of shrinkage is greatly dependent on the composition and texturing of the tissue. For the exact measurement of the biological membrane, quite similar to in vivo, a NIR microscope could be a useful addition to preexisting measurement systems such as caliper, histological measurement, and pachymetric analysis (Schulz et al., 2003). The morphology and orientation of a tissue are quite related to its mechanical and physical properties such as tensile, tear, and optical characteristics (Jalbert et al., 2003).

Analysis of the tissue after histological preparation, including dehydration, embedding, sectioning, and staining, has been much studied using various microscopic imaging and analysis techniques from light microscopy to 
high-voltage electron microscopy (McEwen \& Marko, 1999; Dubey \& Ben-Yehuda, 2011). These microscopes provide high-resolution information on tissue, but it takes a long time to measure, and the value is different from the real value in vivo owing to histological preparation steps. The LEXT system has a good advantage in the analysis of the thickness of some tissues in terms of speed and accuracy close to the real value in vivo, because this excludes any histological preparation steps including dehydration. Some types of confocal microscopes have been applied to analyze the membranous structure such as the cornea and tympanic membrane (Jalbert et al., 2003; Kuypers et al., 2006). The confocal microscope, specially designed for evaluation of the cornea, is widely used in ophthalmology.

The "LEXT" program could reconstruct 3D images by stacking all the internal images for surface rendering, which looked like a square block after scanning (Fig. 2). Each image can be regarded as a virtual section to observe noninvasive transverse sections from the top views in the tissues (Figs. 2, 3). Light attenuation is nearly minimum around the 1,200-1,300-nm wavelength region, as this "optical transparency" barely scatters light from the water constituent within the tissue (Sun et al., 2003).

The virtual sections showed characteristic features according to the nature of the tissues and their depth from the surface to the bottom (Fig. 3). We have some difficulties in interpreting the architectures into classic histological features, as we used routine histology slides stained with hematoxylin and eosin. Nonetheless, it is still difficult to distinguish the tissue from water. Further techniques for improving image contrast are required to enrich the "LEXT" microscopic studies.

In conclusion, our micromorphology measured with the "LEXT" system suggests a great potential in the field of in vivo biomedical research. Also, a further study how a microstructure in the tissue in vivo responds from the external stimuli is possible with a state-of-the-art technique to isolate water artifact from a tissue image. An extensive tissue imaging at real time is quite needed to understand the network integrity of the tissue for defensive function to the environmental changes by absorption and dissipation with distortion, shrinking, and extension.

\section{REFERENCES}

Clay, R.S. \& Court, T.H. (1975). The History of the Microscope: Compiled from Original Instruments and Documents, up to the Introduction of the Achromatic Microscope. London: Holland Press.

Dubey, G.P. \& Ben-Yehuda, S. (2011). Intercellular nanotubes mediate bacterial communication. Cell 144(4), 590-600.

Heinrichs, A. (2009). Stains and fluorescent dyes. Nat Cell Biol 11, S7.

Jalbert, I., Stapleton, F., Papas, E., Sweeney, D.F. \& Coroneo, M. (2003). In vivo confocal microscopy of the human cornea. Br J Ophthalmol 87 (2), 225-236.

Kuypers, L.C., Decraemer, W.F. \& Dirckx, J.J. (2006). Thickness distribution of fresh and preserved human eardrums measured with confocal microscopy. Otol Neurotol 27 (2), 256-264.

Mahapatro, A., Lee, G., Jeong, K. \& Janes, D. (2009). Stable and reproducible electronic conduction through DNA molecular junctions. Appl Phys Lett 95, 083106-3.

McEwen, B.F. \& Marko, M. (1999). Three-dimensional transmission electron microscopy and its application to mitosis research. Methods Cell Biol 61, 81-111.

RusKa, E. (1987). Nobel lecture. The development of the electron microscope and of electron microscopy. Biosci Rep 7, 607-629.

Schulz, D., Iliev, M.E., Frueh, B.E. \& Goldblum, D. (2003). In vivo pachymetry in normal eyes of rats, mice and rabbits with the optical low coherence reflectometer. Vision Res 43, 723-728.

Sun, C.K., Chen, C.C., Chu, S.W., Tsai, T.H., Chen, Y.C. \& Lin, B.L. (2003). Multiharmonic-generation biopsy of skin. Opt Lett 28(24), 2488-2490. 